\title{
Stopping Power Calculations of Compounds by Using Thomas-Fermi-Dirac-Weizsäcker Density Functional
}

\author{
M.Ç. TUFAN* AND H. GÜMÜŞ \\ Department of Physics, Faculty of Sciences and Arts \\ Ondokuz Mayıs University, 55139 Samsun, Turkey \\ (Received February 14, 2008; in final form June 3, 2008)
}

\begin{abstract}
Electronic stopping power of compounds was calculated by using the Thomas-Fermi-Dirac-Weizsäcker density functional. Bragg's rule was employed to determine stopping power of compounds from the elemental stopping power results. Calculations were done for $\mathrm{Be}, \mathrm{B}, \mathrm{O}$, and $\mathrm{Si}$ ions in $\mathrm{Al}_{2} \mathrm{O}_{3}$, $\mathrm{SiO}_{2}$, and $\mathrm{CO}_{2}$ targets by using the Thomas-Fermi-Dirac-Weizsäcker density functional. The obtained results were compared with other ThomasFermi based theoretical calculations and show that using Thomas-FermiDirac-Weizsäcker density functional in stopping power calculations yields reasonably accurate results in especially light systems (with respect to the number of electrons in the system).
\end{abstract}

PACS numbers: $34.50 . \mathrm{Bw}, 34.10 .+\mathrm{x}$

\section{Introduction}

Stopping power, energy loss of energetic particles per unit length in matter, has been studied experimentally and theoretically since the beginning of the 20th century because of its wide areas of application, such as ion implantation, fundamental particle physics, nuclear physics, radiation damage, radiology.

The first quantum mechanical study of stopping power was done by Bethe [1]. Bethe theory of stopping is valid when the projectile's velocity is higher than the Bohr velocity. In Bethe theory, the target is assumed as an elemental material. On the other hand, for compound targets, it is very common to use Bragg's rule [2]. According to this rule, the stopping power of a compound can be calculated by

*corresponding author; e-mail: mctufan@omu.edu.tr 
the linear combination of the stopping powers of the individual elements

$$
S_{\mathrm{e}, \mathrm{C}}=\sum_{i} n_{i} S_{\mathrm{e}, i},
$$

where $S_{\mathrm{e}, \mathrm{C}}$ and $S_{\mathrm{e}, i}$ are the electronic stopping cross-sections of compound and element $i$, respectively, and $n_{i}$ is the molar fraction of element $i$ and such that $\sum_{i} n_{i}=1$.

Although it has been widely applied to compound targets, Bragg's rule is inadequate below the Bethe region of stopping (when projectile's velocity is not high enough for the Bethe approximation to be valid) and also for light projectile-target systems. The deviation of stopping powers in compounds from that estimated with Bragg's rule can be found in a great number of experimental articles and many of these reviewed by Thwaites [3-5]. However, if one accurately calculates the stopping power for elemental targets, obtained results by using Bragg's rule can be more reliable for compounds targets.

In our recent work [6], we calculated stopping power of $\mathrm{Al}_{2} \mathrm{O}_{3}, \mathrm{O}_{2}$, and $\mathrm{SiO}_{2}$ for $\mathrm{O}$ and $\mathrm{Si}$ ions, by using the Thomas-Fermi atomic model and Tietz [7] and Ziegler et al. [8] screening functions. In Ref. [9], we calculated stopping power cross-sections of $\mathrm{C}$ and $\mathrm{Al}$ for $\mathrm{C}, \mathrm{O}$, and $\mathrm{Si}$ ions by using Ziegler's screening function. In our calculations, atomic electronic density plays a key role. In our previous paper [6], we used the Thomas-Fermi (TF) atomic electron density [10,11] with two different screening functions to investigate the effect of screening functions on stopping power. In the present paper, we use a more realistic atomic electron density within the Thomas-Fermi-Dirac-Weizsäcker (TFDW) model [12] to calculate stopping power of compounds.

\section{Calculation of stopping power in the first Born approximation}

In the first Born approximation [13], when a projectile with atomic number $Z_{1}$ and velocity $v$ collides with a stationary target of atomic number $Z_{2}$, the stopping power depending on the number of bound electrons to the projectile, effective charge of target and effective mean excitation energies of both projectile and target is given by [9]:

$$
\begin{aligned}
S_{\mathrm{e}}(v) & =\frac{2 e^{4}}{m_{\mathrm{e}} v^{2}} \\
\times & \times \int_{q_{\min }}^{q_{\max }}\left\{Z_{2}^{*}\left[Z_{1}-{ }_{1} M_{n_{0} n_{0}}(\boldsymbol{q})\right]^{2}+N_{1}\left[Z_{2}-{ }_{2} M_{m_{0} m_{0}}(\boldsymbol{q})\right]^{2}\right\} \frac{\mathrm{d} \boldsymbol{q}}{q^{2}},
\end{aligned}
$$

where $Z_{2}^{*}$ is the number of active target electrons, $N_{1}$ is the number of electrons bound to the projectile, ${ }_{1} M_{n_{0} n_{0}}(\boldsymbol{q})$ and ${ }_{2} M_{m_{0} m_{0}}(\boldsymbol{q})$ atomic form factors of projectile and target, respectively. The projectile-target systems undergo a transition from the electronic state $\left|n_{0}\right\rangle$ for projectile and $\left|m_{0}\right\rangle$ for target to final states $|n\rangle$ and $|m\rangle$, respectively, with energies $E_{n}$ and $E_{m}$. During the collision, the kinetic energy of the projectile is thereby reduced by $\left(E_{n}-E_{n 0}\right)+\left(E_{m}-E_{m 0}\right)=$ $\hbar\left(w_{n n 0}+w_{m m 0}\right)$. Integration limits in Eq. (2) are defined as [14]: 


$$
q_{\min }=\frac{w_{n n_{0}}+w_{m m_{0}}}{v}, \quad q_{\max }=\frac{2 m_{\mathrm{e}} \nu}{\hbar} .
$$

For $q_{\min }$ Cabrera-Trujillo et al. [14] have suggested an applicable average value which is independent of the excited state of the system

$$
q_{\min _{\mathrm{AV}}}=\frac{\left(w_{n n_{0}}+w_{m m_{0}}\right)_{\mathrm{AV}}}{\nu}=\frac{\varepsilon}{\hbar \nu},
$$

and according to $[9] \varepsilon$ is

$$
\varepsilon={ }_{2} I_{0}^{* 1 /(1+\alpha)}{ }_{1} I_{0}^{* \alpha /(1+\alpha)},
$$

where $\alpha$ is [14]:

$$
\alpha=\frac{\left(Z_{2}-Z_{2}^{*}\right)^{2} N_{1}}{\left(Z_{1}-N_{1}\right)^{2} Z_{2}^{*}}
$$

Let us note that $\varepsilon$, given here, is different from that of our previous papers [6,9] because there was written the factor $Z_{1}^{*} / N_{1}$ in the exponential term by mistake.

\section{Determination of the $N_{1}(v), Z_{2}^{*}(v)$ and effective mean excitations energies from the Bohr adiabatic criterion}

To calculate the number of electrons bound to the projectile and the number of active electrons of target, it is convenient to use Bohr adiabatic criterion [15, 16]. According to this theory the electrons whose orbital velocities are lower than the projectile's velocity are stripped from the projectile. Mathematical formulation of this criterion is given by Yarlagadda et al. [17]:

$$
v=b v_{\mathrm{F}}\left(r_{\mathrm{c}}\right)
$$

where $v$ is the velocity of projectile, $v_{\mathrm{F}}(r)=\frac{\hbar}{m_{\mathrm{e}}}\left[3 \pi^{2} \rho(r)\right]^{1 / 3}$ is the Fermi velocity, $\rho(r)$ is the electron density, $b$ is a constant of order of 1.26 and $r_{\mathrm{c}}$ is the critical distance where velocity of electron equals to the velocity of projectile $\left(v_{\mathrm{e}}=v\right)$.

After the calculation of critical distance, the number of electrons bound to the projectile is obtained from

$$
N_{1}=\int_{0}^{r_{\mathrm{c}}} 4 \pi r^{2} \rho(r) \mathrm{d} r
$$

Similarly, by considering symmetry between projectile and target, Sugiyama $[18,19]$ suggested the number of active electrons of target and effective mean excitation energies can be calculated by

and

$$
Z_{2}^{*}=\int_{r_{\mathrm{c}}}^{\infty} 4 \pi r^{2} \rho(r) \mathrm{d} r
$$

$$
\ln I^{*}=\frac{1}{Z^{*}} \int_{r_{b}}^{\infty} \ln \left[\gamma \hbar w_{\mathrm{p}}(r)\right] 4 \pi r^{2} \rho(r) \mathrm{d} r .
$$

Here, $r$ is the distance from the nucleus, $w_{\mathrm{p}}(r)=\left[4 \pi e^{2} \rho(r) / m_{\mathrm{e}}\right]^{1 / 2}$ is the plasma frequency and $\gamma$ is a parameter with value around $\sqrt{2}[20]$. 


\section{Electronic charge density}

Atomic electron density is the key parameter in our stopping power calculation. To calculate the quantities described in the previous section, firstly atomic electron density has to be defined; the more accurate electron density yields the more reliable results.

The Thomas-Fermi electron gas model does not account for the shell structure of the atom. However, it was shown that optimized energies and resulting atomic densities correspond to the Hartree-Fock (HF) values when shellwise electron density is introduced in the TFDW density functional $[21,22]$. It is well known that the Hartree-Fock-Slater wave functions [23] give an accurate representation for the atomic shell structure, however the approximate nature of the present approach does not warrant such a complicated procedure. In present paper, we therefore used the TFDW density functional to determine the electronic charge density.

To account for the shell structure of the atom within the TFDW formalism, we followed the procedure given in Ref. [22]. The atomic electronic density, $\rho$, is written as the sum of orbital densities, $\rho_{i}$ :

$$
\rho=\sum \rho_{i}\left(r, \xi_{i}\right),
$$

where orbital densities are given by

$$
\rho_{i}\left(r, \xi_{i}\right)=N_{i} r^{2 n_{i}-2} \mathrm{e}^{-2 \xi_{i} r} .
$$

In Eq. (12), $n_{i}$ is the principal quantum number of orbital $i, \xi_{i}$ is the variational parameter and relevant normalizing constant $N_{i}$ is defined from the condition

$$
\int \rho_{i}\left(r, \xi_{i}\right) \mathrm{d}^{3} r=w_{i},
$$

where $w_{i}$ is the population of the orbital $i$.

TFDW density functional is given by [21]:

$$
E[\rho]=T[\rho]+V_{\mathrm{ne}}[\rho]+V_{\mathrm{ee}}[\rho],
$$

where $T[\rho]$ is the kinetic energy, $V_{\text {ne }}[\rho]$ is the nuclear-electron attraction energy and $V_{\mathrm{ee}}[\rho]$ is the electron-electron repulsion energy. The first two terms of gradient expansion of kinetic energy [21, 24] is

$$
T[\rho]=T_{0}[\rho]+\lambda T_{\mathrm{w}}[\rho],
$$

where the free electron gas kinetic energy $[10,11]$ is

$$
T_{0}[\rho]=\frac{3}{10}\left(3 \pi^{2}\right)^{2 / 3} \int \rho^{5 / 3} \mathrm{~d} \tau
$$

and the inhomogeneity correction [12] is

$$
T_{\mathrm{w}}[\rho]=\frac{1}{8} \int \frac{\nabla \rho \cdot \nabla \rho}{\rho} \mathrm{d} \tau .
$$

The factor $\lambda$ in Eq. (15) arises from the second-order correction to the gradient expansion of the TF kinetic energy. Although different values of $\lambda$ were suggested by several authors $[24,25]$, we used only $\lambda=1 / 9$ in the present paper. 
The nuclear-electron attraction energy in Eq. (14) is given by

$$
V_{\text {ne }}=-Z \int \frac{\rho}{r} \mathrm{~d} \tau
$$

where $Z$ is the nuclear charge. Besides, electron-electron repulsion energy consists of two terms

$$
V_{\mathrm{ee}}[\rho]=\frac{1}{2} \int \rho \varphi \mathrm{d} \tau+X[\rho] .
$$

The first term in Eq. (24) is classical Coulomb repulsion energy and last term is the Dirac exchange-only energy [26] and it is given by

$$
X[\rho]=-\frac{3}{4}(3 / \pi)^{1 / 3} \int \rho^{4 / 3} \mathrm{~d} \tau .
$$

When the variational representation of the electron density, $\rho\left(\zeta_{1}, \ldots, \zeta_{n} ; r\right)$, is used, Eq. (14) must satisfy the following conditions [21, 27]:

$$
\begin{aligned}
& \frac{\partial E}{\partial \zeta_{i}}=0 \quad(i=1, \ldots n), \\
& \int \rho \mathrm{d} \tau=N,
\end{aligned}
$$

where $\zeta_{i}$ are the variational parameters and $N$ is the total number of electrons in the atom (for neutral atoms $N=Z$ ).

\section{Results and discussion}

In this study electronic stopping cross-sections of $\mathrm{Al}_{2} \mathrm{O}_{3}, \mathrm{SiO}_{2}$ and $\mathrm{CO}_{2}$ were calculated for $\mathrm{Be}, \mathrm{B}, \mathrm{O}$, and $\mathrm{Si}$ ions in the high energy region of stopping. The obtained results were compared with the other results in the literature.

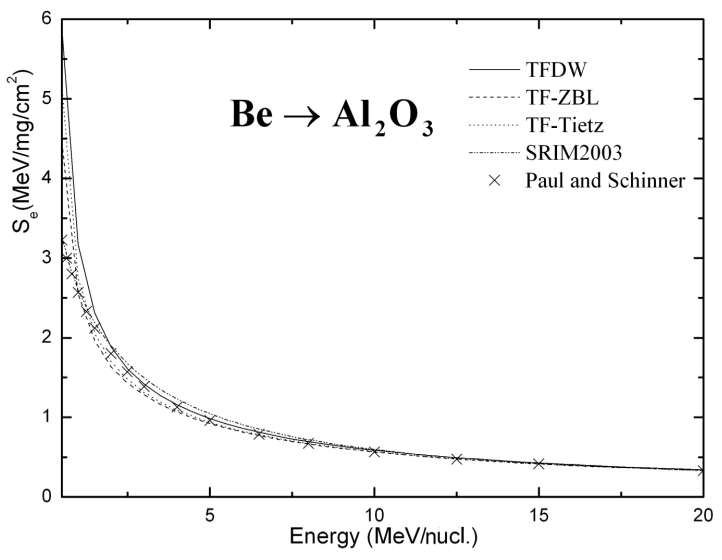

Fig. 1. Electronic stopping power of $\mathrm{Al}_{2} \mathrm{O}_{3}$ for Be ions. The solid line (-) is a plot of stopping powers from Thomas-Fermi-Dirac-Weizsäcker; the dashed line, (- - -) stopping powers from Thomas-Fermi model with Ziegler-Biersack-Littmark's screening function; the dotted line $(\cdots)$ from Thomas-Fermi model with Tietz's screening function; the dashed-dotted-dotted line (-..-) results calculated by SRIM 2003 computer code [29] and the symbols $(\times)$ indicate stopping power values from Paul and Schinner [28]. 


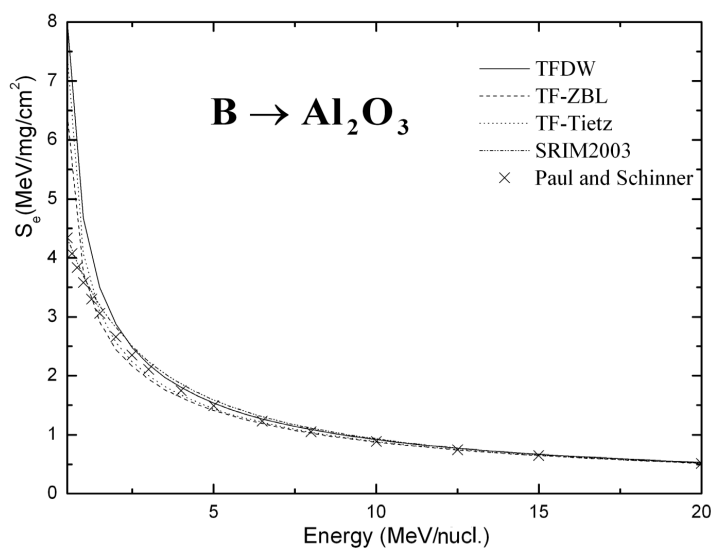

Fig. 2. Electronic stopping power of $\mathrm{Al}_{2} \mathrm{O}_{3}$ for B ions. Symbols and lines are the same as in Fig. 1.

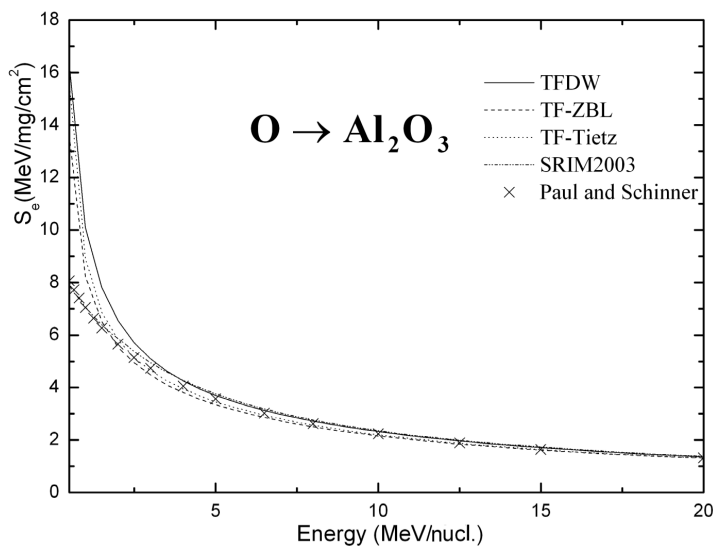

Fig. 3. Electronic stopping power of $\mathrm{Al}_{2} \mathrm{O}_{3}$ for $\mathrm{O}$ ions. Symbols and lines are the same as in Fig. 1.

In present paper, only stopping power results of $\mathrm{Al}_{2} \mathrm{O}_{3}$ are shown in Figs. 1-4 for $\mathrm{Be}, \mathrm{B}, \mathrm{O}$, and $\mathrm{Si}$ ions. In all figures, solid line, dashed line, dotted line represents TFDW results, TF-ZBL, and TF-Tietz results, respectively. TF-ZBL means the calculation by using TF electron density with Ziegler et al.'s screening function [8] while TF-Tietz means the calculation by using TF electron density with Tietz's screening function [7].

As shown from the figures, stopping power results agree with each other at high energies. On the other hand, for energies below the Bethe region deviations are high, which is expected because our calculation is based on the Bethe theory. The reason for these deviations can be explained as in the following: the Bethe theory of the stopping power is in fact valid at high energies, but many scientists 


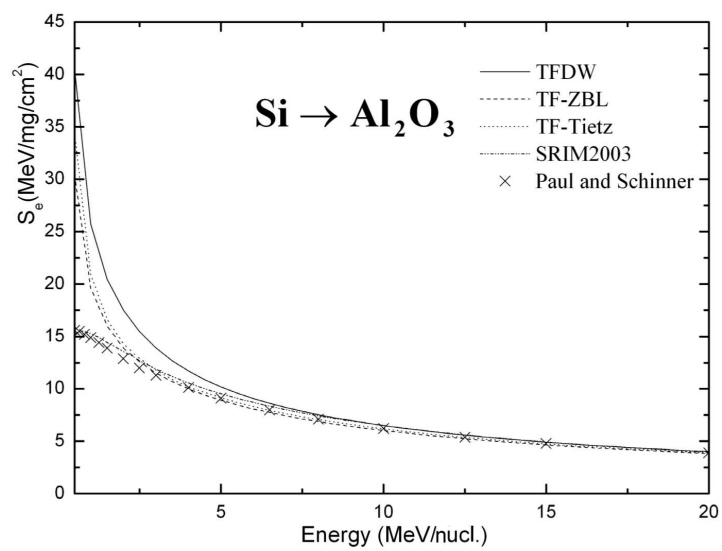

Fig. 4. Electronic stopping power of $\mathrm{Al}_{2} \mathrm{O}_{3}$ for $\mathrm{Si}$ ions. Symbols and lines are the same as in Fig. 1.

TABLE

Percentage deviations of electronic stopping power.

\begin{tabular}{l|c|c|c}
\hline \hline & TFDW & TF-Ziegler & TF-Tietz \\
\hline $\mathrm{Be} \rightarrow \mathrm{CO}_{2}$ & 3.4 & 10.2 & 8.3 \\
$\mathrm{~B} \rightarrow \mathrm{CO}_{2}$ & 3.3 & 10 & 7.8 \\
$\mathrm{O} \rightarrow \mathrm{CO}_{2}$ & 6.4 & 6.1 & 5 \\
$\mathrm{Si} \rightarrow \mathrm{CO}_{2}$ & 11.6 & 6 & 5 \\
\hline $\mathrm{Be} \rightarrow \mathrm{SiO}_{2}$ & 6.1 & 7.9 & 7.2 \\
$\mathrm{~B} \rightarrow \mathrm{SiO}_{2}$ & 7 & 6.8 & 7.2 \\
$\mathrm{O} \rightarrow \mathrm{SiO}_{2}$ & 8.3 & 9.6 & 9.1 \\
$\mathrm{Si} \rightarrow \mathrm{SiO}_{2}$ & 7.5 & 4.6 & 3.2 \\
$(2.5-20 \mathrm{MeV} /$ nucl. $)$ & & & \\
\hline $\mathrm{Be} \rightarrow \mathrm{Al}_{2} \mathrm{O}_{3}$ & 5.1 & 3.8 & 2.9 \\
$\mathrm{~B} \rightarrow \mathrm{Al}_{2} \mathrm{O}_{3}$ & 6.8 & 4.2 & 3.1 \\
$\mathrm{O} \rightarrow \mathrm{Al}_{2} \mathrm{O}_{3}$ & 10.2 & 4.8 & 4.1 \\
$\mathrm{Si} \rightarrow \mathrm{Al}_{2} \mathrm{O}_{3}$ & 20.2 & 6.2 & 6.6
\end{tabular}

have tried to develop this theory to give better results at below this region. The present formalism is based on the Bethe theory, but we modified this theory by considering velocity-dependent number of electrons bound to projectile and active electrons in target. Therefore, we can say that we developed methods of Cabrera-Trujillo and Bethe further to be valid at low energies. Moreover, in our previous papers $[6,9]$ we showed that our calculation method agreed with other theoretical and empirical results better than Bethe's results at energies below high energy region. 
Mean deviations in energies between 1-20 MeV/nucl from Paul and Schinner's results [28] are given in Table for all calculated projectile-target system except $\mathrm{SiO}_{2}$ targets. For $\mathrm{SiO}_{2}$ targets, mean deviations were calculated with respect to SRIM 2003 [29] results at the energy range $2.5-20 \mathrm{MeV} /$ nucl.

\section{Conclusions}

¿From Table, we see that TFDW results are better than TF results in light (with respect to number of electrons in the system) projectile-target systems while $\mathrm{TF}$ results are better for heavy systems. This difference comes from the fact that shell structure of atom, the TFDW model, takes into account the exchange term between electrons which is not taken into account in TF model, and the gradient correction term in kinetic energy. Therefore the electronic charge density obtained by using TFDW model, is real more than that of TF model. However, it is not easy to account for orbital structure of atom especially heavy atoms. Therefore, it is easy to describe heavy atoms with statistical methods like Thomas-Fermi.

The obtained results by using TF model are more reliable for heavy systems because of its statistical nature [6]. In this paper, the stopping power for light systems has been calculated by using TFDW model with electron density describing shell structure of atom. On the other hand, calculations show that it is more convenient to use TF model for heavy systems. Although Bragg's rule is inadequate for light ions and energies outside the Bethe region of stopping, stopping powers of light systems outside the Bethe region were calculated reasonably well.

\section{Acknowledgments}

Authors would like to thank to Prof. Dr. Salvador A. Cruz for his useful discussions and giving his own codes to calculate variation parameters in TFDW model.

\section{References}

[1] H.A. Bethe, Ann. Phys. 5, 325 (1930).

[2] W.H. Bragg, R. Kleeman, Philos. Mag. 10, 318 (1905).

[3] D.I. Thwaites, Nucl. Instrum. Methods Phys. Res. B 12, 84 (1985).

[4] D.I. Thwaites, Nucl. Instrum. Methods Phys. Res. B 27, 293 (1987).

[5] D.I. Thwaites, Nucl. Instrum. Methods Phys. Res. B 69, 53 (1992).

[6] M.Ç. Tufan, Ö. Kabadayı, H. Gümüs, Radiat. Phys. Chem. 76, 631 (2007).

[7] T. Tietz, J. Chem. Phys. 25, 789 (1956).

[8] J.F. Ziegler, J.P. Biersack, U. Littmark, The Stopping and Range of Ions in Solids, Pergamon, New York 1985, p. 321.

[9] M.Ç. Tufan, A. Köroğlu, H. Gümüş, Acta Phys. Pol. A 107, 459 (2005).

[10] L.H. Thomas, Proc. Cambridge Philos. Soc. 23, 542 (1927).

[11] E. Fermi, Mem. Accad. Naz. Lincei 6, 602 (1927). 
[12] C.F. v. Weizsäcker, Z. Phys. 96, 431 (1935).

[13] M. Born, Z. Phys. 38, 803 (1926).

[14] R. Cabrera-Trujillo, S.A. Cruz, J. Oddreshede, J.R. Sabin, Phys. Rev. A 55, 2864 (1997). Erratum: ibid. 59, 4850 (1999).

[15] N. Bohr, Phys. Rev. 58, 654 (1940).

[16] N. Bohr, Phys. Rev. 59, 270 (1941).

[17] B.S. Yarlagadda, J.E. Robinson, W. Brandt, Phys. Rev. B 17, 3473 (1978).

[18] H. Sugiyama, J. Phys. Soc. Jpn. 41, 1339 (1976).

[19] H. Sugiyama, Radiat. Eff. 56, 205 (1981).

[20] J. Lindhard, M. Scharff, Dan. Mat. Fys. Medd. 27, 1 (1953).

[21] W.P. Wang, R.G. Parr, Phys. Rev. A 16, 891 (1977).

[22] S.A. Cruz, C. Diaz-Garcia, A.P. Pathak, J. Soullard, Nucl. Instrum. Methods Phys. Res. B 230, 46 (2005).

[23] E. Clementi, C. Roetti, At. Data Nucl. Data Tables 14, 177 (1974).

[24] W. Yang, Phys. Rev. A 34, 4575 (1986).

[25] Y. Tomishima, K. Yonei, J. Phys. Soc. Jpn. 21, 142 (1966).

[26] P.A.M. Dirac, Proc. R. Soc. Lond. A 112, 661 (1926).

[27] S.A. Cruz, C. Diaz-Garcia, G. Covarrubias, Int. J. Quant. Chem. 102, 897 (2005).

[28] H. Paul, A. Schinner, At. Data Nucl. Data Tables 85, 377 (2003).

[29] J.F. Ziegler, J.P. Biersack, SRIM2003 program, The Stopping and Range of Ions in Matter, version 2003.20, www.srim.org. 